\title{
Characterization of Subscriber Local Loop by Measures and Analysis of Frequency and Impulse Responses
}

\author{
João C. W. A. Costa ${ }^{\mathrm{a}}$, Jacklyn Reis ${ }^{\mathrm{a}}$, Igor Negrão ${ }^{\mathrm{a}}$, Agostinho Castro ${ }^{\mathrm{a}}$, Lamartine V. de Souza ${ }^{* a}$, Klas \\ Ericson ${ }^{\mathrm{b}}$, Fredrik Lindqvist ${ }^{\mathrm{b}}$, Jaume Rius I Riu ${ }^{\mathrm{b}}$ \\ a Applied Eletromagnetism Laboratory - LEA, Federal University of Pará, DEEC, CEP: \\ 66075-900, Belém- PA, Brazil. \\ ${ }^{\mathrm{b}}$ Ericsson AB, Access Signal Processing Laboratory, Älvsjö, Sweden
}

\begin{abstract}
This paper presents methodologies that could be used for characterizing subscriber telephone loops that carry DSL services (ADSL and ADSL2+), by determination and analysis of frequency response, time domain reflectometry, and impulse response of the line. From this analysis, the subscriber loop length, identification and location of impairments such as bridged taps, gauge changes, and open ended termination across the line are carried out. To verify the methodologies presented, results obtained from measurements are drawn and compared to results obtained from computational simulations.
\end{abstract}

Keywords: Loop Qualification, Digital Subscriber Line, Telephone Loop, Time Domain Reflectometry

\section{INTRODUCTION}

The performance of a broadband access service such as Digital Subscriber Loop - DSL technology is directly related to the status of subscriber local loop [1]-[3]. DSL technology could provide a higher data rate than a dial-up connection access, which uses a different frequency band. However, this higher data rate depends on local loop topology. This dependency is due to the fact that the local loop was first designed to Plain Old Telephone System - POTS. A subscriber local loop topology may contain some impairment such as gauge changes, open circuits, bridged taps along the line, and may have a long length which increases the signal attenuation. Such impairments bring about reflections on the main signal; these reflections could be weak or strong ones depend on the nature of the impairments. Furthermore, bridged taps cause strong reflections while gauge changes and open circuit causes weak ones. Additionally, it is easier to identify strong reflection than weak ones. Telephone Companies which provide DSL services should have procedures or tools that characterize and evaluate the capability of a subscriber local loop in carrying DSL signals.

The main focus of this paper is to present methodologies and procedures which could be used by a DSL provider in order to characterize a subscriber local loop, that is estimate its length, detect and locate the presence of open circuits, bridged taps and gauge changes. The subscriber local loop characterization will be based on time frequency response, time domain reflectometry - TDR [4]-[6], and impulse response simulations and measurements. The frequency response measurements consist in carrying out local loop attenuation per tone in DSL frequency band, that is, measurements that start at $4.3125 \mathrm{kHz}$ and finish at $1.104 \mathrm{MHz}$ for Asymmetrical Digital Subscriber Line - ADSL (256 tons) and at 2.208 MHz for ADSL2+ (512 tons) [7]. The impulse response will be performed by Inverse Fast Fourier Transform - IFFT of frequency response obtained from both simulation and measurement. TDR measurements consist in: to send a square wave pulse with specific width along the local loop and analyzing the reflected signal.

The analysis of such results will be used to characterize a subscriber local loop. The nature of the reflection may be perceptible at time and frequency domain furthermore every reflection caused by change of characteristic impedance along the loop contains its signature. This signature will indicate or not presence of such impairments. The total loop length will be calculated by using the time of reflection provided by impulse response or TDR knowing velocity of propagation of the pulse.

*1vsouza@ufpa.br; phone/fax: +55-91-3201-7740; www.ufpa.br/lea 
The paper is organized as follows. In Section 2 is addressed a brief description related to loop qualification issues comprising twisted pair transmission line modeling, primary and secondary parameters, ABCD matrix, transfer function, input impedance, and impulse response determinations, as well as characterization loop, bridged tap analysis, total loop calculation, and gauge changes across the loop. Lab setups for transfer function, input impedance and TDR measurements are discussed in Section 3. The results obtained from computational simulations and measurements are presented and analyzed in Section 4. Finally, in Section 5 the conclusive remarks related to advantages and disadvantage of the methodologies, reached results, and future works are outlined.

\section{LOOP QUALIFICATION}

The status of the subscriber local loop reflects the initial purpose which has been designed, in other words, its construction is related to the needs of old telephone system - POTS. In this manner, some installed elements in local loop aim to improve the quality and the feasibility of the telephone service however these elements may be prejudicial to ADSL service performance. Load coils, bridged taps, transformers, and perhaps gauge changes are some of these elements. Before the ADSL service installation, the telecom operators' wish to obtain information from the subscriber local loop to detect such elements and thus remove them to avoid low ADSL service performance. The more that is known about the local loop, the greater the efficiency of repair crews and less the chance of customer disappointment through installation delays, intermittent failures, or under-performing service. Besides the fast installation guarantee obtainment of this information assures good operation for the service already installed and running.

In this context, loop qualification emerges to test and evaluate the capabilities of subscriber local loop in supporting and maintaining ADSL transmission. There are many test and procedures to perform loop qualification, e.g. measurements in physical layer and computational simulations regarding mathematical models which identify a subscriber loop as a transmission line. The measurements may be performed at Central Office - CO side or in customer's side with the presence of a technician or not, with dedicated measuring devices or ADSL modems or even DSL Access Multiplexers DSLAM. The test when performed only at customer's side or CO side, it is so called Single Ended Line Testing - SELT inasmuch as the test performed at both customer side and CO side is referred by Double Ended Line Testing - DELT.

Performing computational simulations is an important tool to test and evaluate a subscriber local loop which carrying ADSL services. As already discussed, telephone local loop may be modeled as a transmission line, thus Maxwell's equations may be calculated regarding the analysis in frequency and time domain. A brief discussion related to transmission line modeling is addressed as follows.

\subsection{Twisted Pair Transmission Line Modeling}

Twisted pair channel modeling represents an important function in planning and design of ADSL systems. With accurate channel models, predictions based on computational simulations may be carried out regarding good acquaintance of loop transmission potentialities under different condition. The mathematical modeling of a transmission line will be discussed starting with primary parameters, and then secondary parameters and $A B C D$ matrix. After ABCD matrix, the subscriber local loop behavior is investigated in frequency and time domain.

\subsubsection{Primary Parameters}

The primary parameters of a twisted pair transmission line are: Resistance $(R)$, Inductance $(L)$, Capacitance $(C)$, and Conductance $(G)$ which all are variables of frequency. The twisted pair cable is modeled as a transmission line, which in turn can be described with a transmission line equivalent circuit built up with a series resistance $(R)$, a series conductor $(L)$, a parallel capacitance $(C)$ and a parallel conductance $(G)$. For a clearer view of an infinitesimal element $d x$ of a transmission line, see Fig.1 below. The values of the primary parameters are expressed per unit length and are frequency dependent. 


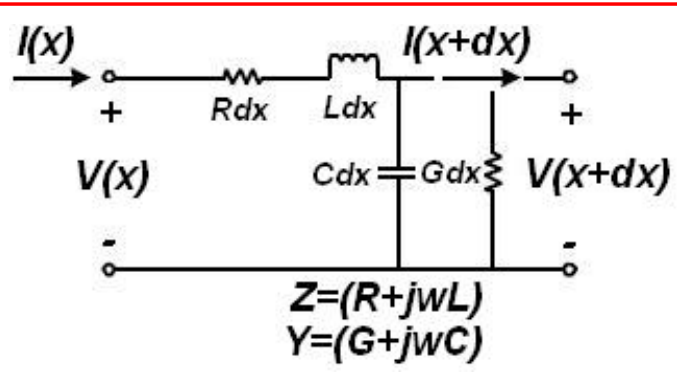

Fig. 1. The transmission line equivalent circuit

A transmission line may be seen by a cascade of these elements. These parameters may be calculated by using the models in the recommendations from the International Telecommunications Union - ITU [8]. Below follows the equations to determine the parameters primary for a certain cable and for a deeper understanding the reader is referred to [1], [2], and [8].

$$
\begin{aligned}
& R(f)=\frac{1}{\frac{1}{\sqrt[4]{r_{0 c}{ }^{4}+a_{c} \cdot f^{2}}}+\frac{1}{\sqrt[4]{r_{0 s}{ }^{4}+a_{s} \cdot f^{2}}}} \\
& L(f)=\frac{l_{0}+l_{\infty} \cdot\left(f / f_{m}\right)^{b}}{1+\left(f / f_{m}\right)^{b}} \\
& C(f)=c_{\infty}+c_{0} \cdot f^{-c_{e}} \\
& G(f)=g_{0} \cdot f^{+g_{e}}
\end{aligned}
$$

\subsubsection{Secondary Parameters}

Now the secondary parameters of a twisted pair transmission line will be derived. The secondary parameters are also called the propagation constant $(\gamma)$ and the characteristic impedance $(Z o)$. Consider an element $d x$ of the transmission line equivalent circuit in Fig. 1. Let the transmission line equivalent circuit be exited with a sinusoidal frequency of $f=\omega / 2 \pi$.

Then let $R, L, C$ and $G$ be the primary parameters per unit length, and functions of frequency. If Kirchhoff's equations are applied to the circuit, the following two differential equations will be obtained:

$$
\begin{aligned}
& \frac{d V}{d x}=-(R+j \omega L) I \\
& \frac{d I}{d x}=-(G+j \omega C) V
\end{aligned}
$$

where $V \equiv V(x, \omega)$ and $I \equiv I(x, \omega)$ are the voltage and current at distance $x$ in the transmission line for the given frequency $f$. The two equations (4) may be rewritten in the following way. 


$$
\begin{aligned}
& \frac{d^{2} V}{d x^{2}}=\gamma^{2} V \\
& \frac{d^{2} I}{d x^{2}}=\gamma^{2} I
\end{aligned}
$$

Where the complex quantity is the propagation constant, which is the first of the secondary parameters.

$$
\gamma(\omega)=\alpha(\omega)+j \beta(\omega) \equiv \sqrt{(R+j \omega L)(G+j \omega C)}=\sqrt{Z \cdot Y}
$$

The quantities per unit length $Z$ and $Y$, impedance and admittance are addressed in Fig.1. As could be seen in (7), the propagation constant is a complex number and may be expressed in terms of $\alpha(\omega)$ and $\beta(\omega)$, which are the attenuation constant and the phase characteristic of the line respectively.

The general steady state solution for the differential equations in (5), are given by

$$
\begin{aligned}
& V(x, f)=V_{0}^{+} e^{-\gamma x}+V_{0}^{-} e^{\gamma x} \\
& I(x, f)=I_{0}^{+} e^{-\gamma x}+I_{0}^{-} e^{-x x}
\end{aligned}
$$

Here $V_{0}^{+}$and $V_{0}^{-}$are the positive and negative going voltage. In a similar way, and $I_{0}^{+}$and $I_{0}{ }^{-}$are the positive and negative going current. By insertion of either of these solutions (8) into the appropriate first order voltage/current differential equation (5) the following will be obtained.

$$
Z_{0}(\omega)=\frac{V_{0}^{+}}{I_{0}{ }^{+}}=-\frac{V_{0}^{-}}{I_{0}^{-}}=\sqrt{\frac{(R+j \omega L)}{(G+j \omega C)}}=\sqrt{\frac{Z}{Y}}
$$

This is equal to the constant characteristic impedance of the twisted pair transmission line. This is the second parameter of the secondary parameters. Please observe that $R, L, C$ and $G$ are still parameters per unit length and frequency dependent. For a deeper explanation of these parameters the interested reader is referred to [1] and [2].

\subsubsection{ABCD Matrix}

A common and practical way to model twisted pair transmission lines is by using the two-port network - 2PN representation. In this representation the electronic circuit in Fig. 1 may be abstracted by the "black box" shown in Fig 2.

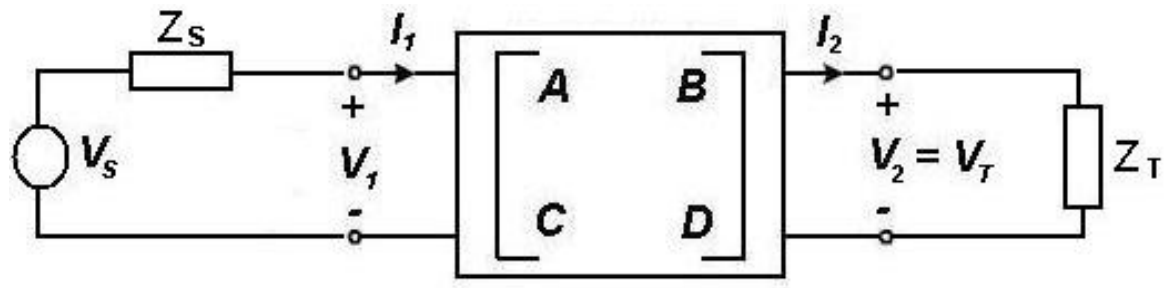

Fig. 2. ABCD matrix representation for a transmission line

The transfer function of any electronic circuit or a twisted pair transmission line may be obtained by the input/output current/voltage relationships in the two-port equivalent. In the Fig. $2 V_{l}$ and $I_{1}$ are the input voltage and current respectively at $x=0 . V_{2}$ and $\mathrm{I}_{2}$ are the output voltage and current at distance $x=d$ across the line respectively.

In this representation, input voltage and current (port 1) and output voltage and current (port 2) relate as follows: 


$$
\begin{aligned}
& {\left[\begin{array}{l}
V_{1} \\
I_{1}
\end{array}\right]=\left[\begin{array}{ll}
A & B \\
C & D
\end{array}\right] \cdot\left[\begin{array}{l}
V_{2} \\
I_{2}
\end{array}\right]=\Phi \cdot\left[\begin{array}{l}
V_{2} \\
I_{2}
\end{array}\right]} \\
& V_{1}=A V_{2}+B I_{2} \\
& I_{1}=C V_{2}+D I_{2}
\end{aligned}
$$

Where $\Phi$ is $\mathrm{ABCD}$ matrix, also called by transmission matrix $\mathrm{T}$ [9], 2x2 order formed by 4 frequency dependent parameters $A, B, C$, and $D$.

In the model, input voltage/current and output voltage/current are related to each other by the so called $\mathrm{ABCD}$ parameters. If a segment of a transmission line of length $d$ has the $V_{T}=V_{d}$ solution $I_{T}=I_{d}$ and thus,

$$
\begin{aligned}
& V_{T}=V(d, f)=V_{0}^{+} e^{-\gamma d}+V_{0}^{-} e^{\gamma d} \\
& I_{T}=I(d, f)=I_{0}^{+} e^{-\gamma d}+I_{0}^{-} e^{\gamma d}
\end{aligned}
$$

Since the two voltage waves in each direction are related to the same direction current waves by the characteristic impedance $\left(Z_{0}\right)$, one can solve the two equations (11) for $V_{0}^{+}$and $V_{0}^{-}$to get:

$$
\begin{aligned}
& V_{0}^{+}=\frac{1}{2}\left(V_{L}+I_{L} Z_{0}(\omega)\right) e^{\gamma d} \\
& V_{0}^{-}=\frac{1}{2}\left(V_{L}-I_{L} Z_{0}(\omega)\right) e^{-\gamma d}
\end{aligned}
$$

By substituting these constants into the general solution (8) and evaluating for the voltage and currents at $x=0$ in terms of those at $\mathrm{x}=\mathrm{d}$, one obtains the following two-port network representation.

$$
\left[\begin{array}{c}
V(0) \\
I(0)
\end{array}\right]=\left[\begin{array}{cc}
\cosh (\gamma d) & Z_{0}(\omega) \sinh (\gamma d) \\
\frac{1}{Z_{0}(\omega)} \sinh (\gamma d) & \cosh (\gamma d)
\end{array}\right] \cdot\left[\begin{array}{c}
V(d) \\
I(d)
\end{array}\right]
$$

The $\mathrm{ABCD}$ parameter modeling is a very useful method when modeling the whole cable section from the $\mathrm{CO}$ to the subscriber. Each section along the transmission path can be represented by its own ABCD matrix. For instance, one $\mathrm{ABCD}$ matrix for the hybrid, one for the transformer, one for bridged taps (if present) and one for the actual cable. The $A B C D$ parameters for the whole transmission path are then obtained by simply cascading all the sections in series.

The ABCD matrix information of a twisted pair transmission line may be easily converted into its input impedance or transfer function. Corresponding calculations can be put into a computer program to generate channel models in conjunction with a few tables of twisted pair cable primary parameters [1] [2] [8].

\subsubsection{Transfer Function, Input Impedance, and Impulse Response}

For a complete two-port network model both a source and terminal impedance has to be added. A complete two-port network is outlined in Fig. 2. The transfer function of a twisted pair telephone loop with source impedance $Z_{S}$ and terminal impedance $Z_{T}$ may then be obtained as follows.

$$
H(s)=\frac{V_{2}(s)}{V_{S}(s)}=\frac{Z_{T}(s)}{Z_{S}(s) \cdot\left(C(s) \cdot Z_{T}(s)+D(s)\right)+A(s) \cdot Z_{T}(s)+B(s)}
$$

And its input impedance may be calculated by: 


$$
Z_{i}(s)=\frac{A(s)+\frac{B(s)}{Z_{T}(s)}}{C(s)+\frac{D(s)}{Z_{T}(s)}}
$$

In which $A, B, C$, and $D$ are the complex frequency dependent ABCD parameters of a twisted pair telephone loop.

The transfer function analyzes the twisted pair telephone loop behavior in frequency domain. However, some practical circumstances are convenient to analyze this behavior in time domain. Such a behavior is important when subscriber loop should be characterized, that is, detection and location of impairments across the loop therefore such impairment has a specific signature in time domain.

Through the transmission line impulse response which characterizes a time invariant linear system it is possible to study the line response for a specific input, that is, it is possible to find the line response for a specific pulse taking into account the satisfied conditions. The line pulse response defined in time domain is the convolution between impulse response and such a pulse. Thus the impulse response for a subscriber local loop is the inverse Fourier transform (IFFT) of frequency response, as follows

$$
h[n]=\mathfrak{I}^{-1}\{H(j \omega)\}
$$

\subsection{Characterization Loop}

This paper aims to present methodology considering the total loop length, detection and location of gauge changes and bridged taps.

\subsubsection{Total Loop Length}

There are many methodologies used to estimate the total subscriber loop length in the literature, e.g. TDR test which consist in sending a known pulse across the line and analyze its reflection [4]. In [10] the total loop length is calculated by using the transfer function slope regarding the linear trend in its slope.

Our methodology consists in applying the IFFT in the transfer function provided by measurements or simulations thus the impulse response for the transmission line is obtained. With impulse response curve, the round trip travel time of impulse is identified then multiplied by its velocity of propagation which is estimated around $66 \%$ of velocity of light Error! Reference source not found. The round trip travel time is caused by the termination mismatch between the line characteristic impedance and termination impedance, in our case $100 \Omega$.

\subsubsection{Bridged Tap Detection}

To detect and locate a bridged tap the loop transfer function is also used [10]. In this research, the appearance of periodic notching in the transfer function indicates the presence of a bridged tap in the loop and in [3] and [11] the reader may understand how the first notch frequency or null is used to estimate the length of the bridged tap. When a single bridged tap is present in the loop, the notches in the transfer function appear at frequencies where the length of the bridged tap equals an odd multiple of one quarter of the wavelength.

To detect the presence of a bridged tap in telephone loop, we are going to use a methodology similar to calculate the total loop length. The impulse response is carried out by IFFT of transfer function, then the reflections caused by bridged tap, the open circuit termination of the bridged tap, and the bridged tap caused by termination impedance are going to be used to calculate the location of the bridged tap, the bridged tap length, and the total loop length respectively.

\subsubsection{Gauge Changes}

Telephone cables are usually deployed following the regular gauging design rules, i.e., cables near the $\mathrm{CO}$ have a thinner diameter inasmuch as cables near the customer home have a thicker diameter. Thinner cables have higher characteristic impedance than thicker ones, thus a signal that encounters this discontinuity passes from a medium with higher impedance to a medium with lower impedance. In general, when a signal travels on a loop and passes from a section with higher characteristic impedance to a section with a lower one, the reflection will always be negative. And vice versa; when a signal travels on a loop and passes from a section with lower characteristic impedance to a section with a higher one, the echo will always be positive. 
When the objective is to detect echoes reflections such as caused by gauge changes or even caused by far discontinuities, the methodology regarding the IFFT of transfer function to calculate the impulse response seems to be inefficient. This is due to the fact that the methodology does not take into account the distributed RLCG behavior of the loop. If the reflection is very strong such as caused by a bridged tap or near discontinuity, this behavior may be neglected, while in the case of weak reflections, this approach turns out to be too harsh.

In [4] a method is presented to detect a gauge change present in the loop. Such a technique is based on the slowly decaying signal subtraction from the main signal that enters in local loop. To perform such method a priori information related to the first loop section is required. The extension for such a technique is presented in [5] [6] where no priori information is required and it may be applied to a loop formed by more than two gauge changes. In our cause, we are going to use this technique however no priori information will be passed to the algorithm; the approach will consider the first loop section always started with $0.40 \mathrm{~mm}$ gauge cable. The results will show that such approach provides good results to locate gauge changes present in the loop.

\section{LAB SETUPS}

To execute the measurements of transfer function, input impedance and time domain reflectometry related this paper, the test setups are addressed in Fig.3. As could be seen, to perform transfer function measurements, setup drawn in Fig.3 (a) was used, in such a test setup we are not going to investigate the difference in transfer function curve provided by a loop terminated with and without a splitter filter, we are going to omit the modem + telephone + splitter filter and take into account only a terminal impedance of $100 \Omega$ (Balun). On the other hand, in Fig.3 (b) is given the setup which will be used to perform input impedance and TDR measurements of loop open circuit terminated. The test loops are going to be the European loops \# 2, \# 5, and \# 8 from ITU recommendation G.996.1 [8].

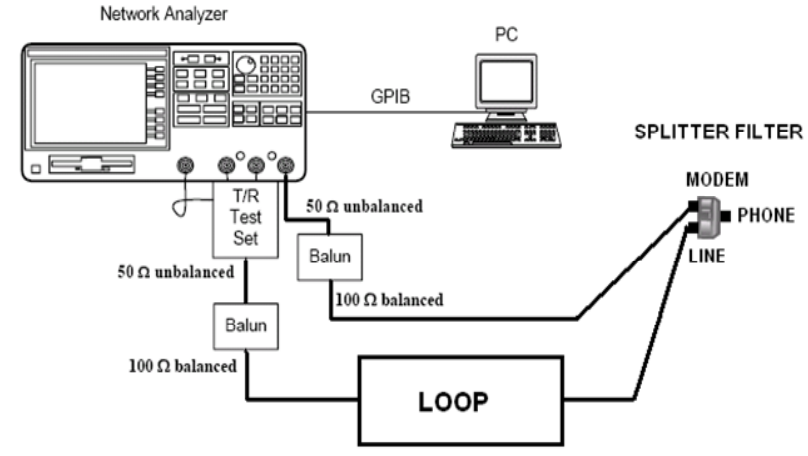

(a)

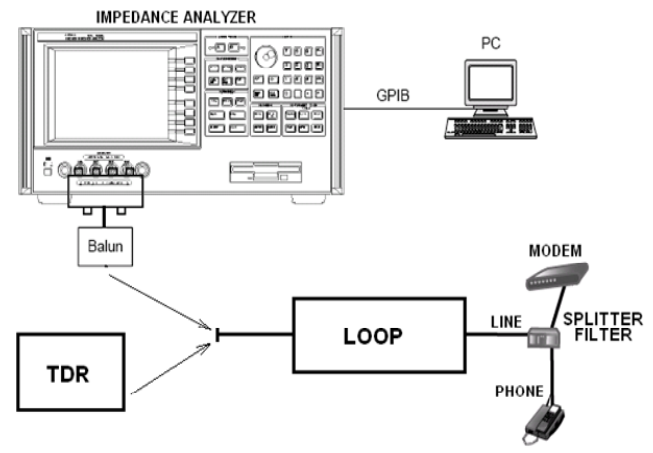

(b)

Fig. 3. (a) Test setup used to perform transfer function measurements. (b) Test setup used to perform input impedance and TDR measurements.

\section{RESULTS AND ANALYSIS}

This section presents the results obtained from measurements and computational simulations. Each scenarios will be analyzed taking into account its transfer function, input impedance, impulse response, and TDR how were explained before.

In the following results, the transfer function obtained from simulations is compared to the obtained from measurements for test loops number 2, 5, and 8 as well as input impedance and impulse response. TDR test is only applied to test loop number 5 because it is formed by a mixture of different gauge cables. 


\subsection{ETSI 2}

As could be seen in the result bellow, no reflections were detected to ETSI loop 2 because is single loop, i.e., it is formed by only one gauge cable $(0.50 \mathrm{~mm})$. The curves results obtained from measurements and simulations follow the same fashion for transfer functions, input impedance, and impulse response. The total loop length may be calculated by using the reflection point detected in Fig 4. (c) $\left(1.698^{*} 10^{-5} \mathrm{~s}\right)$; multiplying this time by the velocity of propagation (about $66 \%$ of $c$ ) the total loop length is $3378 \mathrm{~m}$ (absolute error of $0.64 \%$ ). The input impedance comes to around $100 \Omega$, the value agrees completely with the result presented in the literature for typical telephone transmission lines.

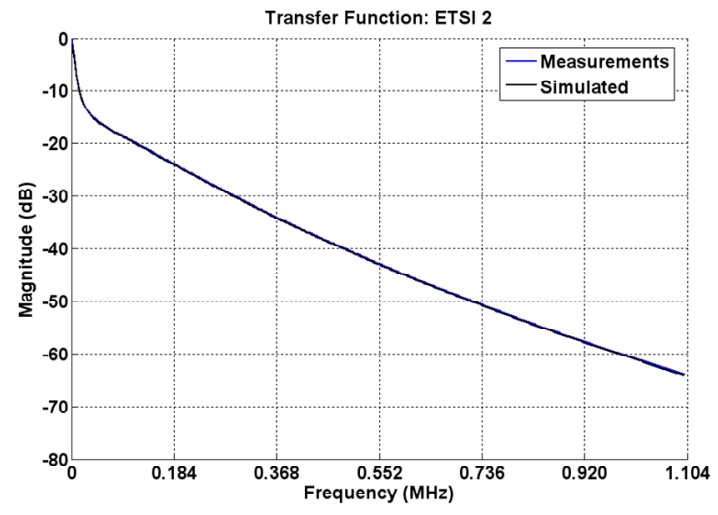

(a)

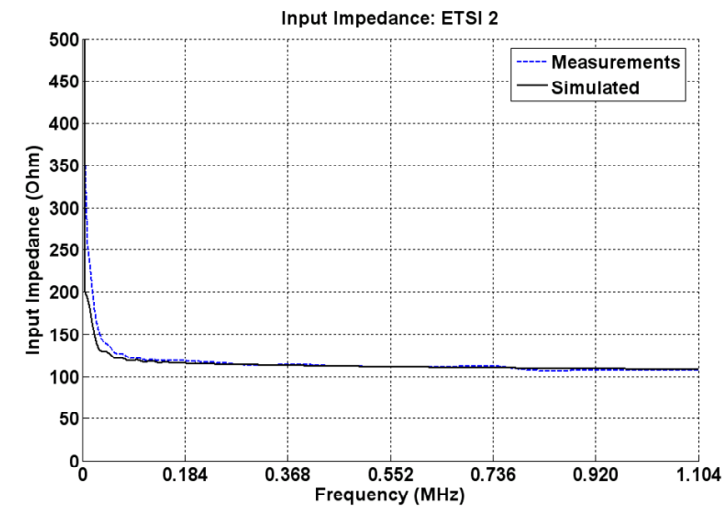

(b)

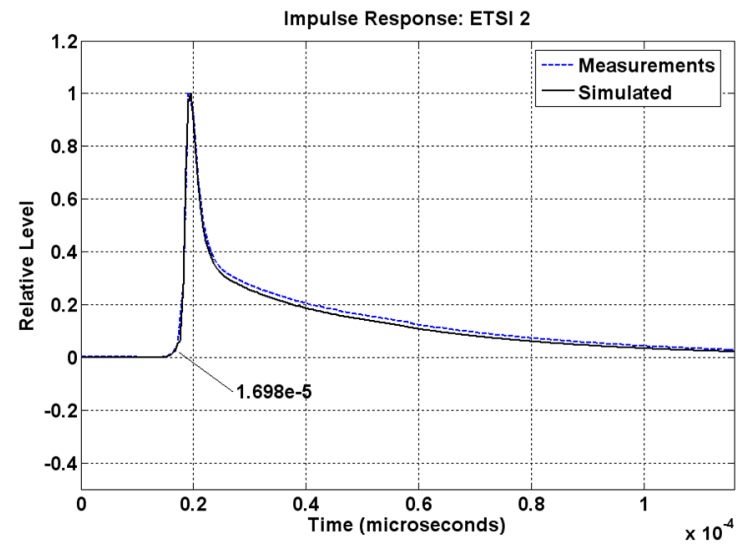

(c)

Fig. 4. (a) Transfer function for ETSI 2. (b) Input impedance for ETSI 2. (c) Impulse response for ETSI 2.

\subsection{ETSI 5}

As could be seen in the result above, no reflections were detected to ETSI loop 5 even such a loop is not single loop, i.e., it is formed by a mixture of gauge cables $(0.40 \mathrm{~mm}, 0.50 \mathrm{~mm}, 0.63 \mathrm{~mm}$, and $0.90 \mathrm{~mm})$. The curves results obtained from measurements and simulations follow the same fashion for transfer functions, input impedance, and impulse response. The total loop length may be calculated by using the reflection point detected in Fig 5. (c) $\left(1.59 * 10^{-5} \mathrm{~s}\right)$; multiplying this time by the velocity of propagation the total loop length is $3180 \mathrm{~m}$ (absolute error of $0.62 \%$ ). 


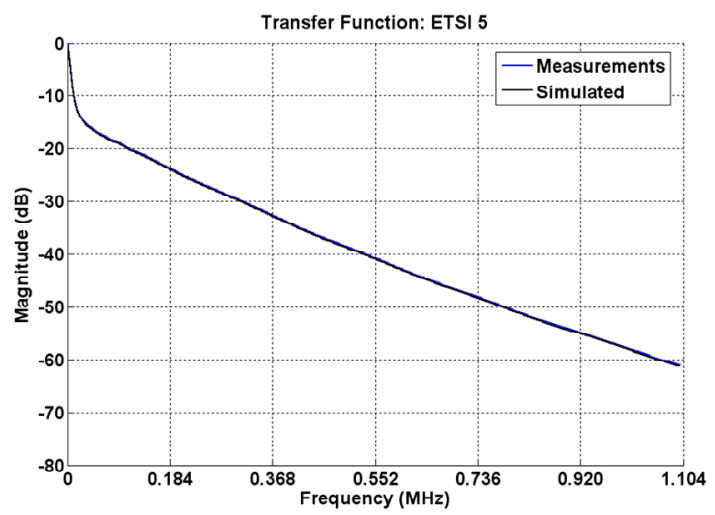

(a)

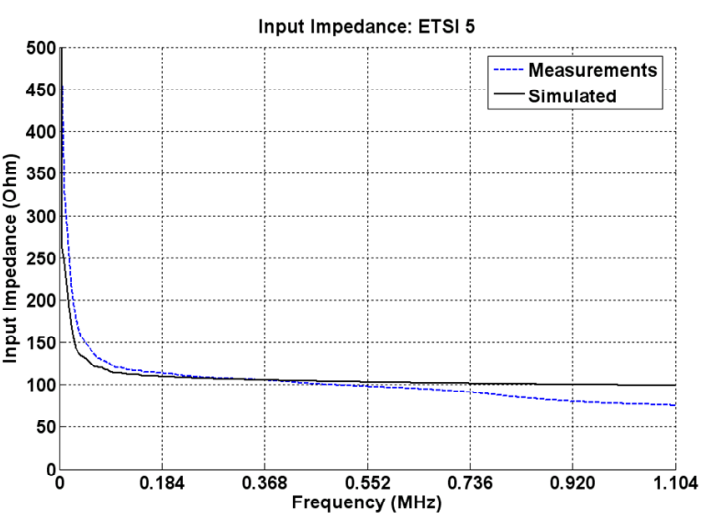

(b)

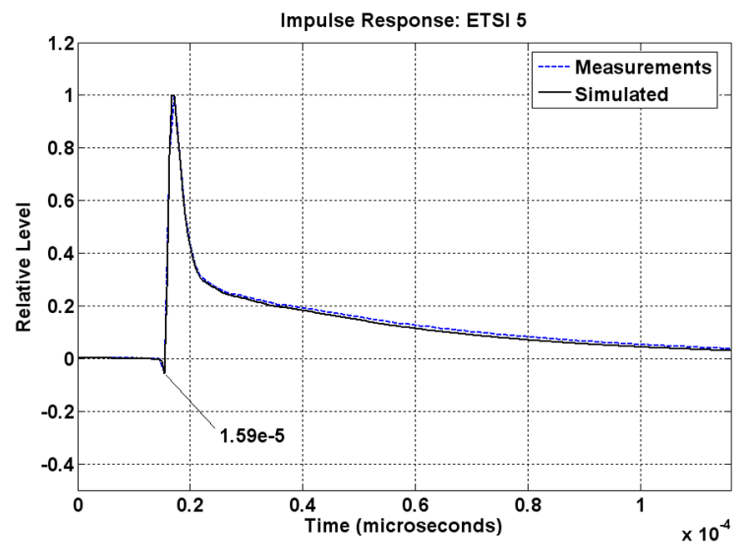

(c)

Fig. 5. (a) Transfer function for ETSI 5. (b) Input impedance for ETSI 5. (c) Impulse response for ETSI 5.

The gauge changes were not detected using transfer function and impulse response however the notches seen in the input impedance indicates that mismatches are present in the loop. Thus, to detect such a weak mismatches caused by the gauge changes in the loop the subtraction technique may be applied. TDR test was performed to obtain the reference curve for a loop with $0.40 \mathrm{~mm}$ gauge and length of $7.5 \mathrm{~km}$. The result is outlined in Fig 6 where the reflection points are identified and may be used to locate such reflections caused by gauge changes in $1440 \mathrm{~m}$ (error of $0.68 \%$ ), $2220 \mathrm{~m}$ (error of $0.90 \%$ ), and $2800 \mathrm{~m}$ (error of $3.70 \%$ ).

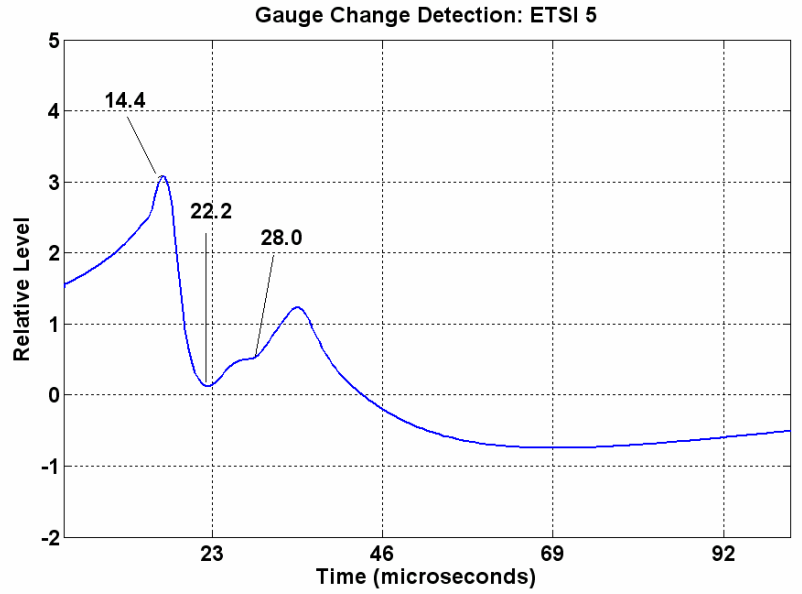

Fig. 6. Improved TDR to detect gauge changes for ETSI 5. 


\subsection{ETSI 8}

Test loop number 8 has two bridged taps as could be seen in Fig 7, however according to the best knowledge's author it is not possible to identify the effect for a single bridged tap using transfer function and impulse response analysis. Using the method explained before, the point marked in the graphic in Fig. 7 (a) may be used to calculate the bridged tap length $(500 \mathrm{~m})$. Using impulse response in Fig. 7 (c), the points marked in the curve give the location of the bridged tap $\left(8.152 * 10^{-5}\right)$ and its length $\left(1.54 * 10^{-5}\right.$ and $\left.2.038^{*} 10^{-5}\right)$. The bridged tap length is calculated by subtracting $1.54 * 10^{-5}$ from $2.0381 * 10^{-5}$ and multiplying by the pulse velocity then gives $498 \mathrm{~m}$ (error of $0.4 \%$ ). The bridged tap location is calculated by the multiplying the first reflection $\left(8.152 * 10^{-5}\right)$ by the pulse velocity and then subtracting the bridged tap length twice gives $634.4 \mathrm{~m}$ (error of $15.4 \%$ ); .

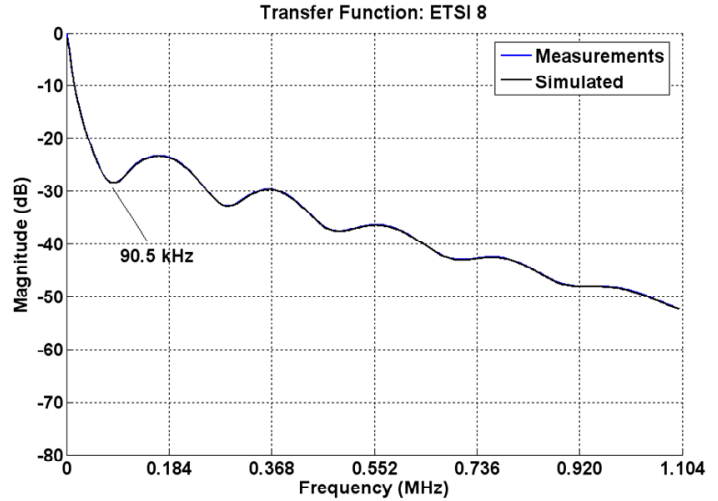

(a)

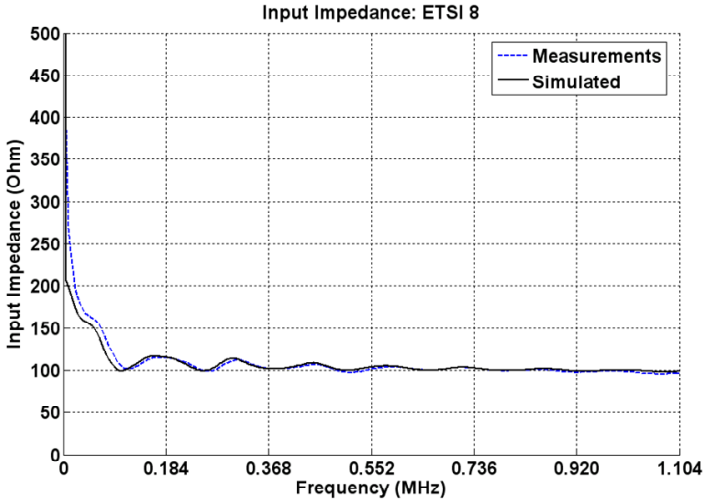

(b)

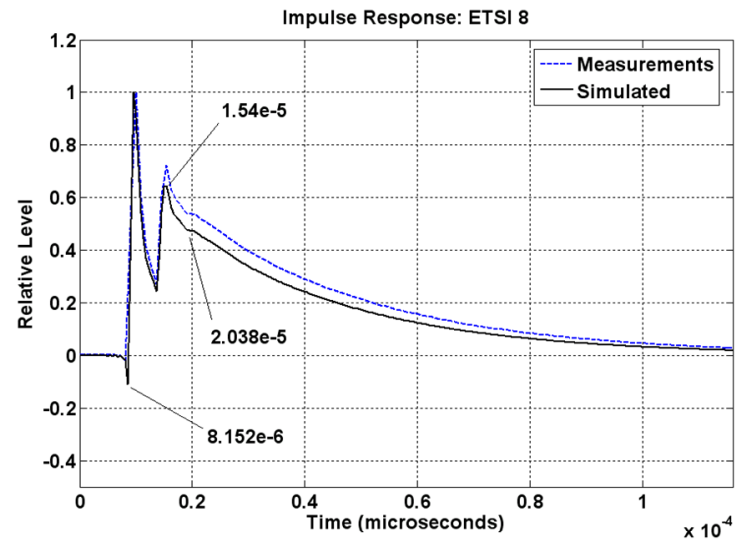

(c)

Fig. 7. (a) Transfer function for ETSI 8. (b) Input impedance for ETSI 8. (c) Impulse response for ETSI 8.

\section{CONCLUSIONS}

This paper has presented methods to carry out the total loop length, bridged tap detection and location as well as gauge changes detection using an improved TDR technique. Such methodologies may be used by ADSL service provider in order to characterize the subscriber loop and guarantees the high quality of the service. The methods may be applied both using measurements and computational simulations from transmission line mathematical models thus comparative analysis may be performed to validated the method. 


\section{ACKNOWLEDGMENT}

Authors would like to thank the Research and Development Centre, Ericsson Telecomunicações S.A., Brazil, Federal University of Pará, Department of Electrical and Computing Engineering, Belém, Pará, Brazil. And Ericsson AB, Access Signal Processing Laboratory, Älvsjö, Sweden. Some of the authors want to acknowledge the financial support received from the European Commission IST 6th Framework and from the Swedish Agency for Innovation Systems, VINNOVA, through the IST - MUSE and the Eureka - Celtic BANITS projects respectively, which partially enabled this work.

\section{REFERENCES}

1. T. Starr, J. M. Cioffi, and P. J. Silverman, Eds., "Understanding Digital Subscriber Line Technology”. New York: Prentice Hall, 1999.

2. W. Y. Chen, "DSL - Simulation Techniques and Standards Development for Digital Subscriber Line Systems", Indianapolis, Macmillan Technical Publishing.

3. J.-J.Werner, "The HDSL Environment", IEEE Journal on Selected Areas in Communications, Vol. 9, NO. 6, August 1991.

4. S. Galli, and D. L. Waring, "Loop Makeup Identification Via Single Ended Testing: Beyond Mere Loop Qualification”, IEEE Journal On Selected Areas in Communications, Vol. 20, NO. 5, June 2002.

5. S. Galli, and K. J. Kerpez, "Single-Ended Loop Make-Up Identification-Part I: A Method of Analyzing

TDR Measurements”, IEEE Transactions on Instrumentation and Measurement, Vol. 55, NO. 2, April 2006.

6. K. J. Kerpez, and S. Galli, "Single-Ended Loop- Makeup Identification-Part II: Improved Algorithms and Performance Results", IEEE Transactions on Instrumentation and Measurement, Vol. 55, No. 2, April 2006.

7. Ericsson AB. "Line Testing”, Ethernet DSL White Paper, 2004.

8. International Telecommunication Union - Telecommunication G.996.1, Test Procedures for Digital Subscriber Line (DSL) Transceivers, 2001.

9. S. Galli, "Exact Conditions for the Symmetry of a Loop", IEEE Communications Letters, Vol. 4, NO. 10, October 2000.

10. J. L. Fang, C. Zeng, and J. Cioffi, "Bridged Tap Location Estimation", Electrical Engineering Department, Stanford University, California, USA.

11. G. H. Im, and J. J. Werner, "Effect of Bridged Taps at VDSL Frequencies”, Lucent Technologies, September 1997, Minneapolis. 